\title{
Motor execution affects action prediction
}

\author{
Anne Springer ${ }^{\mathrm{a}, \mathrm{b}, *}$, Simone Brandstädter ${ }^{\mathrm{a}}$, Roman Liepelt ${ }^{\mathrm{a}, \mathrm{c}}$, Teresa Birngruber $^{\mathrm{a}}$, Martin Giese ${ }^{\mathrm{d}}$, \\ Franz Mechsner ${ }^{\mathrm{e}}$, Wolfgang Prinz ${ }^{\mathrm{a}}$ \\ ${ }^{a}$ Max Planck Institute for Human Cognitive and Brain Sciences, Leipzig, Germany \\ ${ }^{\mathrm{b}}$ University of Potsdam, Exzellenzbereich Kognitionswissenschaften, Department of Sport and Exercise Psychology, Potsdam, Germany \\ ' Westfälische Wilhelms University, Department of Psychology, Junior Group 'Neurocognition of Joint Action', Münster, Germany \\ ${ }^{\mathrm{d}}$ Hertie-Institute for Clinical Brain Research and Centre for Integrative Neuroscience, University Clinic Tübingen, Tübingen, Germany \\ e Northumbria University, Newcastle upon Tyne, UK
}

\section{A R T I C L E I N F O}

Article history:

Accepted 15 March 2011

Available online 8 April 2011

\section{Keywords:}

Action prediction

Internal simulation

Motor execution

Common coding

Perception-action-link

\begin{abstract}
A B S T R A C T
Previous studies provided evidence of the claim that the prediction of occluded action involves real-time simulation. We report two experiments that aimed to study how real-time simulation is affected by simultaneous action execution under conditions of full, partial or no overlap between observed and executed actions. This overlap was analysed by comparing the body sides and the movement kinematics involved in the observed and the executed action. While performing actions, participants observed point-light (PL) actions that were interrupted by an occluder, followed by a test pose. The task was to judge whether the test pose depicted a continuation of the occluded action in the same depth angle. Using a paradigm proposed by Graf et al., we independently manipulated the duration of the occluder and the temporal advance of the test pose relative to occlusion onset (occluder time and pose time, respectively). This paradigm allows the assessment of real-time simulation, based on prediction performance across different occluder time/pose time combinations (i.e., improved task performance with decreasing time distance between occluder time and pose time is taken to reflect real-time simulation). The PL actor could be perceived as from the front or back, as indicated by task instructions. In Experiment 1 (front view instructions), evidence of action simulation was obtained for partial overlap (i.e., observed and performed action corresponded either in body side or movement kinematics), but not for full or no overlap conditions. The same pattern was obtained in Experiment 2 (back view instructions), ruling out a spatial compatibility explanation for the real-time pattern observed. Our results suggest that motor processes affect action prediction and real-time simulation. The strength of their impact varies as a function of the overlap between observed and executed actions.
\end{abstract}

(c) 2011 Elsevier Inc. All rights reserved.

\section{Introduction}

Human mirror neuron research has indicated that perceiving an action activates similar cortical networks as executing the same action (Gallese, Fadiga, Fogassi, \& Rizzolatti, 1996; for a review see Iacoboni \& Dapretto, 2006). This corresponds to the notion that perception and action share common representational codes (Hommel, Müsseler, Aschersleben, \& Prinz, 2001; Prinz, 1990, 1997). Numerous behavioral studies have supported this notion by showing that action execution affects action observation and vice versa (for a review see Schütz-Bosbach \& Prinz, 2007). For instance, visual motions were detected less efficiently when they went in the same direction as concurrently performed actions

\footnotetext{
* Corresponding author at: University of Potsdam, Exzellenzbereich Kognitionswissenschaften, Department of Sport and Exercise Psychology, Am Neuen Palais 10, D-14469 Potsdam, Germany.

E-mail address: anne.springer@uni-potsdam.de (A. Springer).
}

(Hamilton, Wolpert, \& Frith, 2004; Zwickel, Grosjean, \& Prinz, 2007). On the other hand, movement execution was faster when go-stimuli displayed the to-be-executed movements (Brass, Bekkering, \& Prinz, 2001; Craighero, Bello, Fadiga, \& Rizzolatti, 2002), and the variance of executed movements was increased while observing a different movement relative to the same movement (Kilner, Paulignan, \& Blakemore, 2003).

The relationship between perception and action has been described in terms of 'representational overlap' (e.g., Dinstein, Hasson, Rubin, \& Heeger, 2007; Marconi, Pecchioli, Koch, \& Caltagirone, 2007). According to the theory of event coding (TEC), codes of perceived events and planned actions share a common representational domain. As a consequence, perceptual codes and action codes may prime each other on the basis of their representational overlap in this domain (Hommel et al., 2001). For instance, during different motor cognitive tasks (i.e., motor imagery; movement observation), the cortical representations of a target muscle and a functionally related muscle were enhanced within a single task and across different 
tasks, indicating a topographical and functional overlap of motor cortical representations (Marconi et al., 2007).

Representational overlap may be used for anticipating others' action by mapping those actions onto one's own motor repertoire (Gallese, 2005; Jeannerod, 2001). Humans seem to run internal sensorimotor simulations when predicting the future course of observed actions (see Sebanz \& Knoblich, 2009; Wilson \& Knoblich, 2005, for reviews). Flanagan and Johansson (2003) showed that eye movements during action observation were predictive rather than reactive, and Kilner, Vargas, Duval, Blakemore, and Sirigu (2004) found that motor-related brain activity occurred prior to observing a predictable movement. Furthermore, increased premotor activation was found when observers predicted the future course of partly invisible action sequences (Stadler et al., in press), indicating that internal simulation is used for action prediction.

\subsection{The present research}

Although motor representations of observed actions may be involved in predicting these actions, this issue has not directly been investigated. The present study aimed to test the impact of motor processes on action prediction. Based on studies showing a close link between perception and action (Brass et al., 2001; Craighero et al., 2002; Hamilton et al., 2004; Kilner et al., 2003, 2004; Liepelt, von Cramon, \& Brass, 2008; Stadler et al., in press; Zwickel et al., 2007), we suggest that motor processes are involved in internal action simulation. More specifically, the degree of a representational overlap between executed and observed movements (i.e., the degree to which they share common representational features) should modulate the degree to which simulation processes are recruited during an action prediction task (Hommel et al., 2001).

We examined how motor execution affects the performance in a visual action prediction task that is considered to reflect internal action simulation. We used a paradigm by Graf et al. (2007), who presented videos of a point-light (PL) actor performing familiar actions that were briefly occluded and then immediately continued by the presentation of a static test pose. This test pose was either in the same orientation as the previously seen action or was slightly rotated in depth. Participants judged whether or not it showed a coherent continuation of the previously seen action (i.e., whether the action phase depicted in the test pose had the same or a different depth of orientation). The authors' basic assumption was that the last visible action segment before occlusion is internally updated in real time during occlusion. Two factors were manipulated. First, the 'pose time', defined as the time that had passed between the last visible action frame before occlusion and the test pose shown after occlusion, was varied (i.e., pose times of 100, 400 and 700 ms after occlusion onset). Second, and independently, the 'occluder time', defined as the time gap that elapsed between the last visible action frame and the actual presentation of the test pose, was manipulated (i.e., occluder times of 100,400 and $700 \mathrm{~ms}$ ).

According to the real-time hypothesis, performance should be best when pose time (PT) and occluder time (OT) correspond to each other, because in this case, the internal action representation (updated in real time) should match the actual test pose. In addition, performance should decrease as the time distance between both factors increases. The larger this difference, the more difficult it should be to assess whether or not the test pose shows a coherent continuation of the action sequence. Thus, real-time simulation predicts an interaction between PT and OT that takes the form of a 'monotonic distance function': Performance should be best when the temporal distance between OT and PT is zero, intermediate at a distance of $\pm 300 \mathrm{~ms}$ (100/400; 400/700; 400/
$100 ; 700 / 400)$ and lowest at a distance of $\pm 600 \mathrm{~ms}(100 / 700$ and $700 / 100)$.

Indeed, previous results (Graf et al., 2007; Springer \& Prinz, 2010) showed that performance was best when occluder time and pose time corresponded and decreased with increasing time distance, supporting the notion that action prediction involves internal real-time processes. This notion corresponds to the view that sensorimotor simulations enable the extrapolation of future actions (Grush, 2004; Thornton \& Knoblich, 2006). Efficient body control requires the estimation of one's own body state prior to movement execution, which is based on internal forward models. These forward models predict the sensory consequences of movements in real time by relying on motor commands (i.e., efference copies) (Wolpert \& Flanagan, 2001). They may also be applied when predicting the actions observed in another individual (Blakemore \& Frith, 2005; Kilner, Friston, \& Frith, 2007).

To test our assumption that the processes involved in an action prediction task pertain to a motor representational format, we adapted the paradigm by Graf et al. (2007) by adding a concurrent motor execution task. While watching arm movements of a point-light (PL) figure, the participants executed arm movements synchronized in time to the PL movements (see Fig. 1). We used three different conditions. In the 'full overlap' condition, both the executed actions and the observed actions were fully congruent and involved the same anatomical body side and the same movement pattern. In contrast, in the 'partial overlap' condition, executed and observed actions differed either in the anatomical body side used or in the exact movement pattern involved. In the 'no overlap' condition, observed and executed actions were fully incongruent, involving different anatomical body sides and different movement patterns (as specified below). As the two dimensions - which we refer to as 'body side' and 'movement pattern', respectively - varied independently, this design allowed us to systematically test how the degree of a representational overlap between executed and observed actions affects internal action simulation (indicated by a monotonic distance function).

In short, we expected to observe a monotonic distance function, indicating real-time simulation. Observers should show best performance when the test pose shown after the occlusion is temporally coherent with the assumed internal real-time model of that pose (real-time hypothesis). Second, we expected the monotonic distance function to be modulated by action execution (motor modulation hypothesis). More specifically, when executed and observed actions fully correspond (full overlap condition), the need to internally simulate observed actions should be reduced, because the executed action may itself provide an internal reference for the match with the external test pose (Wilson \& Knoblich, 2005). Hence, internal simulation would not provide any information that goes beyond the information which is available from the executed movement itself, and simulation would be redundant.

Conversely, in the 'no overlap' condition, the information available from action execution is entirely different from the observed action in two dimensions. Hence, the motor representations used for motor execution may cause interference, thus impeding internal simulation (Prinz, 1997; Wilson \& Knoblich, 2005). Indeed, the cost/benefit ratio for running internal simulations should be more balanced for a partial representational overlap (Hommel et al., 2001). Here, simulation may be partially efficient for solving the task (i.e., monotonic distance function), whether this is possibly due to congruence in terms of the anatomical body sides used (Wilson \& Knoblich, 2005) or the exact movement patterns involved (Kilner et al., 2003). In sum, these cost/benefit considerations suggest no simulation for full and zero overlap (for different reasons, however) but simulation for the partial overlap conditions. 


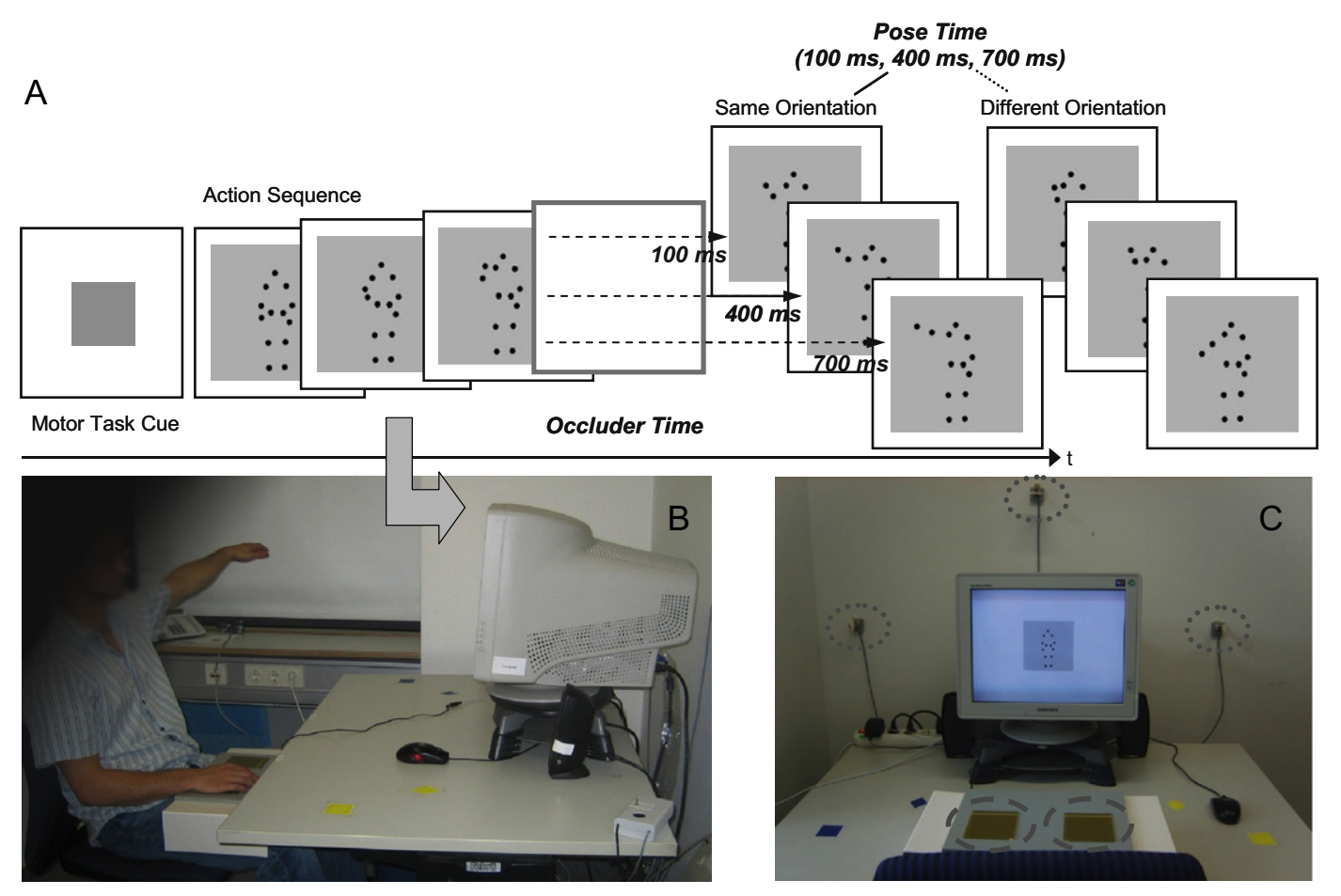

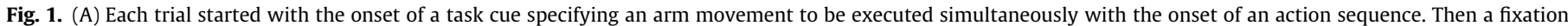

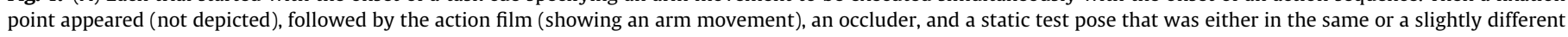

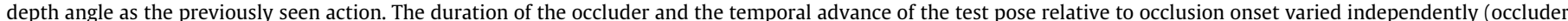

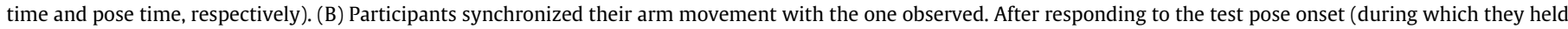

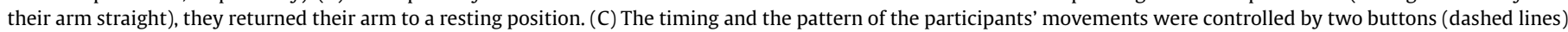
and three sensors (dotted lines), respectively.

\section{Experiment 1}

\subsection{Participants}

Thirty-five participants (17 female, age rage $20-31$; mean age 24.7) took part in this experiment. All were right-handed according to self-report (Oldfield, 1971), German native speakers, and had normal or corrected-to-normal vision. Participation was voluntary and paid.

\subsection{Stimuli and design}

Each trial started with a symbolic task cue (onset $1200 \mathrm{~ms}$ ) at the screen centre specifying an arm movement which had to be executed starting with the beginning of the point-light (PL) action. After the task cue, a central black fixation dot (1200 ms) occurred, followed by the PL action sequence depicting a person performing an arm movement. After some time, an occluder was presented, immediately followed by a static test pose showing a continuation of the arm movement either in the same direction as in the action sequence prior to occlusion or a different direction (i.e., slightly rotated towards the observer) (Fig. 1). The participants were instructed to judge whether or not the test pose showed a coherent continuation of the previously seen movement at any point in time (i.e., whether the arm movement was continued in the same direction or slightly rotated in depth).

The duration of the action sequences in the test phase varied from 1254 to $1782 \mathrm{~ms}$ (38-54 frames). The test pose was presented until a response was given or for a maximum of $2500 \mathrm{~ms}$. If participants did not respond within $2500 \mathrm{~ms}$ after test pose onset, the trial was aborted. Nevertheless, feedback (300 ms) about response accuracy was provided after every test pose. The next trial started $500 \mathrm{~ms}$ after the response (or trial abortion). Participants were in- structed to respond as quickly and as accurately as possible by pressing a foot pedal ('yes'/'no') with the right or left foot, respectively. Four independent variables were used within participants: (1) the body side of motor execution (i.e., anatomically same or different to the PL movement), (2) the executed movement pattern (i.e., same or different to the PL movement), (3) the occluder time (i.e., occluder duration of 100,400 or $700 \mathrm{~ms}$ ) and (4) the pose time (i.e., the time gap between the last visible frame before occlusion and the test pose shown after occlusion, again corresponding to 100,400 or $700 \mathrm{~ms}$ ) (Fig. 1).

Four arm movements were shown, performed by a PL character: reaching out the right arm to the right side; lifting the right arm upwards over the head; reaching out the left arm to the left side; lifting the left arm upwards over the head (cf. Fig. 1). We used point-light stimuli (Johansson, 1973) since they emphasize motion information while minimizing the feasibility of response strategies related to other cues. The stimuli were recorded from a righthanded female agent in front view using a motion capture system with seven cameras (Vicon Motion Systems Ltd., Oxford, UK) with a temporal sampling rate of $120 \mathrm{~Hz}$. Trajectory data were processed using commercial software by VICON and further manipulated in MATLAB.

Each point-light display consisted of 13 black dots on a grey background, located at the major joints of the actor's body (centre of the head, shoulders, elbows, wrists, sternum, and centre of pelvis, knees, and ankles). The dots were approximately $5 \mathrm{~mm}$ in diameter. The actions were rendered with $30 \mathrm{~Hz}$. The point-light actor was about $9 \mathrm{~cm}$ in height and moved within an area of 340 pixels width and 340 pixels height (about $12 \mathrm{~cm} \times 12 \mathrm{~cm}$ ) at the centre of the screen. An occluder of the same size was rendered in white with a light green frame.

The stimuli entailed one distractor pose for every correct test pose, created by depth-rotating the correct arm posture slightly to- 
wards the observer (thus demanding a 'different' response to the test pose due to the change in the visual angle after the occlusion). Rotation was based on the fitting of a three-dimensional kinematic model to the arm and manipulating the shoulder angle. A rotation angle of $40^{\circ}$ was chosen for every action and pose time, respectively. Note that the 'different' trials entail two error sources: a temporal error (when occluder time and pose time diverge) and an additional spatial error (due to depth rotation of the arm posture shown in the test stimulus). Since there is no definite hypothesis about the interaction of these two error sources, clear-cut predictions for the 'different' trials cannot be derived. Therefore, following the procedure by Graf et al. (2007), our analyses focused on 'same' trials only (i.e., trials demanding a 'same' response due to a continuous depth orientation of the action phase depicted in the test pose). These 'same' trials allowed us to investigate the temporal factor of interest in its neat form (Graf et al., 2007), as 'same' trials do not imply an additional spatial error, thus avoiding any confounds. More specifically, the real-time hypothesis predicts an interaction between the occluder time and the pose time, with best performance when both correspond to each other (see Fig. 1).

During each PL action trial, the participants carried out one of the above-described arm movements. They were carefully instructed to synchronize their movement with the observed one (i.e., to synchronize the onset and speed of movement) to ensure motor activation lasted throughout the experimental trial. At the onset of the static test pose, participants held their arm constantly stretched out at full length (sideways or upwards, respectively), yielding a flat trajectory irrespective of the actual duration of the occlusion. This procedure allowed us to avoid the possibility that their movement impeded their response. After the response to the test pose was given (via foot pedals), they had to move their arm down to a resting button (Fig. 1). No feedback was given to the motor execution task.

The timing and the order of the participants' movements were carefully controlled within each trial, as illustrated in Fig. 1. Two hand sensors, installed on a board the participants held on their lap, recorded the time point of movement onset and offset. Three additional photoelectric beam sensors, placed on the wall in front of the participants, monitored the pattern of their movement (heading upwards vs. heading sideways; cf. Fig. 1C). This procedure enabled us to identify trials in which the participants failed to perform the instructed movement, and also trials in which they did not successfully synchronize with the observed movement (e.g., started earlier or later than the PL actor, or finished before the response to the test pose was given). Only the trials in which the movements were performed correctly with respect to time synchrony, body side and movement pattern entered the analyses.

\subsection{Procedure}

The experiment was conducted in a dimly lit, quiet room. It comprised two sessions (each lasting approximately $1.5 \mathrm{~h}$ ) with a break of $3 \mathrm{~h}$ between them. At the beginning, participants were instructed that the point-light character displayed a person from the front view (i.e., a person looking towards them). This was followed by a familiarization, a practice and a test phase, all including all action stimuli.

The test phase included 288 trials per session (yielding a total of 576 trials) which were administered in 12 blocks (each comprising 24 trials); each block included a constant occluder time $(100,400$, or $700 \mathrm{~ms}$ ). The trials were randomly chosen from all possible stimulus combinations ( 2 body sides $\times 2$ movement patterns $\times 3$ pose times $\times 3$ occluder times $\times 2$ responses). All motor conditions and all pose time/occluder time combinations appeared equally often; identical motor conditions did not succeed each other. There was a self-timed break after every block. The initial familiarization showed each point-light action once and in full length. Afterwards, the two tasks were practiced separately (12 trials per task), followed by 48 practice trials randomly chosen from all possible stimulus combinations, in which motor execution and the action prediction task were done in parallel. During this practice, the participants extensively practised synchronizing their own movements with the observed one (as explained above) and were encouraged to ask if they had any questions about the tasks and procedure.

Participants sat approximately $82 \mathrm{~cm}$ from a Samsung Sync master 997 MB 19-inch colour monitor (resolution of $1024 \times 768$ pixels; refresh rate of $90 \mathrm{~Hz}$ ) controlled by 'Presentation'. Since the distractors (i.e., the depth rotated arm poses) were highly similar to the correct test poses, the overall task difficulty was high. Accordingly, the data analysis focused on the error rates. Reaction times were analyzed for correct responses only. Analyses of variance (ANOVAs) for repeated measurements were used, with degrees of freedom corrected according to the Huynh-Feldt formula (Huynh \& Feldt, 1970). Post-hoc paired comparisons ( $t$-test, twotailed) were Bonferroni corrected.

\section{Results and discussion}

First, we expected that the factors occluder time and pose time would interact, producing a monotonic distance function (i.e., realtime simulation hypothesis). Second, we expected that the monotonic distance function would be influenced by motor execution. More specifically, the monotonic distance function should vary according to the degree to which executed and observed movements correspond in the anatomical body side used and the exact movement pattern involved, thus yielding conditions of 'full' vs. 'partial' vs. 'no' representational overlap (i.e., motor modulation hypothesis). The results are provided in Appendix A.

Error rates were entered into - a repeated measures analysis of variance (ANOVA) with the within-subjects factors occluder time (100 vs. 400 vs. $700 \mathrm{~ms}$ ), pose time (100 vs. 400 vs. $700 \mathrm{~ms}$ ), body side (congruent vs. incongruent) and movement pattern (same vs. different). The results revealed a highly significant interaction of occluder time $\times$ pose time, $F(4,136)=5.53, M S E=.022, p<.001$, in line with the real-time hypothesis. Furthermore, a significant main effect of pose time was indicated, $F(2,68)=96.66$, $M S E=.310, p<.001$, due to substantially increased error rates for short pose times of $100 \mathrm{~ms}(43.7 \%, S E=3.1)$ than for longer pose times (400 ms: $7.5 \%, S E=1.2 / 700 \mathrm{~ms}: 8.6 \%, S E=1.5$ ). This increase can be attributed to the fact that the PL arm position shown in the $100 \mathrm{~ms}$ test poses, by definition, only advanced a little (i.e., by $100 \mathrm{~ms}$ ) and was thus still close to the PL actor's body. As a consequence, these test poses were more difficult to judge regarding the spatial angle of the PL arm as compared to the pose times where the PL arm was stretched out further away from the body (i.e., 400 and $700 \mathrm{~ms}$ ). Thus, $100 \mathrm{~ms}$ pose times were excluded from all further analyses to avoid the possibility that mere stimulus-driven effects overlay the critical occluder time/pose time interactions.

An additional four-way ANOVA (repeated measures) revealed an interaction of occluder time $\times$ pose time $\times$ body side $\times$ movement pattern, $F(2,68)=6.67, M S E=.010, p<.004$, while the occluder time $\times$ pose time interaction did not reach significance, $F(2,68)=2.12, M S E=.013, p=.14$; n.s. Importantly, there was neither an occluder time $\times$ pose time $\times$ body side interaction, $F(2,68)=.159, M S E=.101, p=.85$; n.s., nor an occluder time $\times$ pose time $\times$ movement pattern interaction, $F(2,68)=.801, M S E=.008$, $p=.45$; n.s. This finding indicates that neither the body side itself nor the movement pattern itself affected the critical occluder time/pose time conditions. Furthermore, no main effects of pose 
time, $F(1,34)=.688, \quad M S E=.033, \quad p=.41 ; \quad$ n.s., body side, $F(1,34)=.682, \quad M S E=.010, \quad p=.42 ; \quad$ n.s., and occluder time, $F(2,68)=2.03, M S E=.016, p=.14$; n.s., were indicated. The main effect of movement pattern was significant, $F(1,34)=6.03$, $M S E=.019, p<.02$, due to higher error rates for different movement patterns (i.e., observed and executed movement patterns diverged) than for same movement patterns $(9.21 \%, S E=1.4$ vs. $6.87 \%, S E=1.1$, respectively).

However, our motor modulation hypothesis predicts that the occluder time $\times$ pose time interaction (i.e., real-time simulation) is modulated by the degree of a representational overlap between executed and observed movements. Because executed and observed movements differed in two dimensions (i.e., anatomical body side, movement pattern) and because these two dimensions varied independently, three conditions of representational overlap emerged. In the 'full overlap' condition, executed and observed movements involved the same anatomical body side and the same movement pattern. In the 'partial overlap' condition, executed and observed movements differed either in the anatomical body side used or the movement pattern involved. In the 'no overlap' condition, observed and executed movements differed in both, the body side and the movement pattern.

To test the effects of a representational overlap, a three-way repeated measures ANOVA including the within-subjects factors occluder time ( 100 vs. 400 vs. $700 \mathrm{~ms}$ ), pose time (400 vs. $700 \mathrm{~ms}$ ) and representational overlap (full vs. partial vs. no overlap) was run. Most importantly, it revealed a significant three-way interaction, $F(4,136)=2.83, M S E=.007, p<.03$, consistent with our motor modulation hypothesis. No main effects of pose time, $F(1,34)=.93$, $M S E=.027, p=.34$; n.s., occluder time, $F(2,68)=1.61, M S E=.012$, $p=.21$; n.s., or representational overlap, $F(2,68)=1.76$, $M S E=.010, p=.18$; n.s., were indicated, and there were no further interaction effects.

More specifically, the real-time hypothesis predicts that performance is best when occluder time and pose time correspond to each other. In addition, performance should deteriorate with increasing time distance between both factors (due to increased deviance of the actual test pose from the internally generated real-time model), yielding a monotonic distance function. To test this monotonic distance function, we averaged the error rates over the same absolute distance levels between occluder time (OT) and pose time (PT) (i.e., 0, 300 and $600 \mathrm{~ms}$ ) (Graf et al., 2007). For example, an OT of $400 \mathrm{~ms}$ and a PT of $700 \mathrm{~ms}$ result in a (positive) distance of $+300 \mathrm{~ms}$, while an OT of $700 \mathrm{~ms}$ and a PT of $400 \mathrm{~ms}$ result in a (negative) distance of $-300 \mathrm{~ms}$; both cases were referred to as 'distance $300 \mathrm{~ms}$ '. It should be noted that the positive time distances (i.e., the pose time exceeded the occluder time) $(8.55 \%$, $S E=1.3$ ) and the negative time distances (i.e., the occluder time exceeded the pose time) $(8.21 \%, S E=1.6)$ did not differ from each other. This was confirmed by an additional three-way repeated measures ANOVA with the within-subjects factors distance direction (positive vs. negative), body side (congruent vs. incongruent) and movement pattern (same vs. different) indicating neither a main effect of distance direction, $F(1,34)=.234, M S E=.014$, $p=.63$; n.s., nor any interaction with this factor.

In line with the real-time hypothesis, the errors (i.e., misses) increased monotonically with increasing time distance between occluder time and pose time, thus yielding a monotonic distance function. A three-way ANOVA (repeated measures) with the within-subjects factors distance ( 0 vs. 300 vs. $600 \mathrm{~ms}$ ), body side (congruent vs. incongruent) and movement pattern (same vs. different) revealed a significant main effect of distance, $F(2,68)=4.04$, $M S E=.017, p<.04$, exhibiting a monotonic trend, $F(1,34)=5.47$, $M S E=.015, p<.03$, in the predicted way. There was a significant interaction of distance $\times$ body side $\times$ movement pattern, $F(2,68)=5.08, M S E=.005, p<.01$. However, no interaction of dis- tance $\times$ body side, $F(2,68)=1.11, M S E=.007, p=.33$; n.s., and no interaction of distance $\times$ movement pattern, $F(2,68)=.78$, $M S E=.011, p=.41 ; n . s$., were indicated. These findings speak against the alternative that either a correspondence in the body side or a correspondence in the movement pattern, by itself, influenced the monotonic distance function (i.e., internal simulation).

If this is indeed the case, this leads to the question of what varied the function. Our motor modulation hypothesis predicts that the degree of a representational overlap between executed and observed movement matters. To test this hypothesis, we ran an additional ANOVA (repeated measures) with the within-subjects factors distance ( 0 vs. 300 vs. $600 \mathrm{~ms}$ ) and representational overlap (full vs. partial vs. no overlap). The results confirmed a significant interaction between 'distance' and 'representational overlap', $F(4,136)=2.65, M S E=.005, p<.05$, in line with the motor modulation hypothesis. Furthermore, there was a marginal main effect of distance, $F(2,68)=3.35, M S E=.014, p<.06$, with a marginal monotonic trend, $F(1,34)=3.37, M S E=.013, p<.07$, in the predicted way. No main effect of representational overlap was indicated, $F(2,68)=2.27, M S E=.008, p=.12$; n.s.

Additional separate analyses confirmed a significant monotonic distance effect for the 'partial overlap' condition, $F(2,68)=6.17$, $M S E=.004, p<.005$. This monotonic distance effect exhibited a significant linear trend, $F(1,34)=11.17, M S E=.004, p<.002$, in correspondence to the motor modulation hypothesis. In addition, no such monotonic distance function occurred for the conditions 'full overlap', $F(2,68)=.63$, MSE $=.007, p=.51$; n.s., and 'no overlap', $F(2,68)=3.24, M S E=.012, p=.06$; n.s. Hence, these findings provide evidence of our view that the degree of a representational overlap between executed and observed movements varied the monotonic distance function (i.e., internal, simulation). More specifically, in contrast to the 'full' and 'no' overlap conditions, a 'partial overlap' seemed to favour internal real-time simulation (as indicated by a monotonic distance function); whether this is possibly due to congruence in the anatomical body sides used or the exact movement patterns involved.

Analogous to the errors, the reaction times (RTs) were entered into a repeated measures ANOVA with the within-subjects factors occluder time ( 100 vs. 400 vs. $700 \mathrm{~ms}$ ), pose time ( 400 vs. $700 \mathrm{~ms}$ ), body side (congruent vs. incongruent) and movement pattern (same vs. different). In line with the real-time hypothesis, the results showed a significant occluder time $\times$ pose time interaction, $F(2,68)=5.27, M S E=4384.64, p<.008$. In addition, there was a significant main effect of occluder time, $F(2,68)=6.60$, $M S E=11329.34, p<.002$, due to higher RTs for short occlusion durations of $100 \mathrm{~ms}(M=710.41 \mathrm{~ms}, S E=26.26)$ relative to longer occlusions $\quad(400 \mathrm{~ms}: \quad M=710.41 \mathrm{~ms}, \quad S E=27.33 / 700 \mathrm{~ms}$ : $M=700.19 \mathrm{~ms}, S E=25.57)$. This RT increase may be attributed to short response preparation times elapsing between action offset and test pose onset for short occlusion durations of $100 \mathrm{~ms}$, as response preparation may be decreased for 'early' test stimuli which are presented earlier than on average (Bertelson \& Tisseyre, 1968; Graf et al., 2007; Sanders, 1998).

To test the monotonic distance function (taken to reflect realtime simulation), a three-way repeated measures ANOVA with the within-subjects factors distance (0 vs. 300 vs. $600 \mathrm{~ms}$ ), body side (congruent vs. incongruent) and movement pattern (same vs. different) was run. The results revealed a highly significant main effect of distance, $F(2,68)=11.36, M S E=6574.76, p<.001$, exhibiting a linear trend, $F(1,34)=15.78, M S E=7366.67, p<.001$, in the predicted way (i.e., increased RTs with increasing time distance between occluder time and pose time). Thus, a monotonic distance function was also reflected in the RTs (see Appendix A). An additional within-subjects ANOVA with the factors distance $(0$ vs. 300 vs. $600 \mathrm{~ms}$ ) and representational overlap (full vs. partial vs. no overlap) confirmed a main effect of distance, 
$F(2,68)=12.67, M S E=4092.18, p<.001$, with a significant linear trend, $F(1,34)=18.30, M S E=4868.85, p<.001$, in the predicted way. No further RT effects were obtained. Therefore, no speedaccuracy-trade-offs were indicated (i.e., the RT pattern was not inverse to the error pattern).

Overall, the results revealed the predicted monotonic distance function (indicating real-time simulation). Moreover, in line with the motor modulation hypothesis, the distance function was modulated by overt motor execution. A pronounced monotonic distance function emerged when executed and observed movements were similar (but not identical) to the PL actor's movements (i.e., 'partial overlap'). In contrast, the function was absent for 'full overlap' and 'no overlap' (i.e., when both movements involved the same body sides and movement patterns or, on the other hand, involved different body sides and movement patterns, respectively). These results suggest that the degree of a motor representational overlap influenced the monotonic distance function (taken as a signature of real-time simulation). In other words, the function was neither affected by a congruence of the anatomical body sides itself nor by a congruence of the movement patterns itself. This finding corresponds to our idea that the degree of representational overlap between executed and observed movement matters (i.e., motor modulation hypothesis).

It should be noted that we use the term 'full overlap' from an anatomical point of view (i.e., executed and observed movements involved the same movement pattern and the same anatomical body side). Alternatively, one may refer to spatial correspondence (when executed and observed movements involved the same movement pattern and spatially congruent body sides, i.e., the same side of the screen). In Experiment 1, this constellation was present in the 'partial overlap' condition (i.e., 'different body side, same movement pattern', implying a 'mirror-inverted' constellation). This condition showed the monotonic distance function.

In fact, previous research suggests that spatial correspondence matters, too. For instance, movements were executed faster when go-signals displayed the end position of these movements in a mirror-inverted way (Craighero et al., 2002). Using a visual detection task, Christensen, Ilg, Karnath, and Giese (2009) found that the visual detection of others' motion was modulated in a spatially selective manner by one's own activated motor representations. Furthermore, the attenuation of beta cortical oscillations in MEG was found to vary depending on the side of the screen on which an action was observed (Kilner, Marchant, \& Frith, 2009), indicating that motor influences on perception may be due to spatial congruence rather than anatomical mappings between executed and observed actions.

With respect to the present data, one may alternatively assume that spatial congruence increased the likelihood with which our participants engaged in internal simulation, thus showing a pronounced monotonic distance function in the condition of 'partial overlap' (including spatial correspondence).

The aim of Experiment 2 was to directly test this alternative. Therefore, in contrast to Experiment 1, participants in Experiment 2 were instructed that they would see the PL actor's rear (i.e., back view), while all other experimental parameters were kept constant. Under front view conditions (Experiment 1), spatial and anatomical body side congruence is dissociated. In contrast, the back view manipulation (Experiment 2) implies that spatial and anatomical body side congruence correspond directly. Hence, if the PL action and the executed action involve the same anatomical body side (e.g., left arm), they occur on the same side of the screen (e.g., left side). If spatial congruence between executed and observed movements matters, a monotonic distance function is expected to emerge under the condition of a full representational overlap.

\section{Experiment 2}

\subsection{Participants}

Thirty right-handed participants took part in the experiment (17 female, age rage 20-31; mean age 24.8). All were German native speakers and had normal or corrected-to-normal vision. Participation was voluntary and paid.

\subsection{Design and procedure}

The identical stimuli, tasks and procedure as in Experiment 1 were used, except for the instructions regarding viewing direction, as explained above.

\subsection{Viewing direction manipulation check}

An independent pre-test of the point-light (PL) stimulus material was conducted to ensure that the actions, originally filmed from in front of the actor, could equally likely be perceived as displaying a person from the front or from the back. This is a precondition to explicitly instructing participants that either the front or the back view of the (identical) PL figure was shown. Sixteen independent volunteers (all right-handed; 8 female) watched a randomly generated sequence including all PL actions in full length (i.e., without occlusion) yielding a total of 160 trials. Each action was presented in coherent and incoherent (i.e., slightly rotated) depth orientation, yielding a total of twenty times per condition. The raters had to indicate whether they believed that the PL actor was shown from the front or from the back by pressing a corresponding key after each action sequence. The results showed that the (identical) PL figure was rated as being shown from the front and from the back with equal probability (49.80\%:50.20\% of all trials; i.e., not above chance level), indicating that the PL stimuli were ambiguous with respect to front vs. back view and thus suitable for instruction-based viewing direction manipulations.

\section{Results and discussion}

Error rates were entered into a four-way repeated measures ANOVA with the within-subjects factors occluder time (100 vs. 400 vs. $700 \mathrm{~ms}$ ), pose time ( 100 vs. 400 vs. $700 \mathrm{~ms}$ ), body side (congruent vs. incongruent) and movement pattern (same vs. different). The results revealed the critical interaction of occluder time $\times$ pose time, $F(4,116)=4.62, M S E=.021, p<.004$, as predicted by the real-time hypothesis. Again, a highly significant main effect of pose time was obtained, $F(2,58)=82.27, M S E=.311$, $p<.001$, indicating increased error rates for $100 \mathrm{~ms}$ pose times $(45.0 \%, S E=3.8)$ than for longer pose times ( $400 \mathrm{~ms}: 10.3 \%$, $S E=1.6 / 700 \mathrm{~ms}: 9.9 \%, S E=1.5$ ). Thus, following the procedure of Experiment 1, the pose times of $100 \mathrm{~ms}$ were excluded from all further analyses. The results of all occluder time/pose time conditions are provided in Appendix A.

An additional four-way within-subjects ANOVA revealed an interaction of occluder time $\times$ pose time $\times$ body side $\times$ movement pattern, $F(2,58)=6.40, M S E=.009, p<.003$. Importantly, analogous to Experiment 1, there was no interaction of occluder time pose time $\times$ body side, $F(2,58)=1.75, M S E=.009, p=.18$; n.s., and no interaction of occluder time $\times$ pose time $\times$ movement pattern, $F(2,58)=.46, M S E=.011, p=.63$; n.s. Hence, neither the factor body side alone nor the factor movement pattern alone seemed to have an impact on the critical occluder time/pose time conditions (corresponding to our hypothesis that the degree of a representational overlap in body side and movement pattern matters). No further interactions and no main effects of pose 
time, $F(1,29)=.14, \quad M S E=.021, p=.72 ;$ n.s., and body side, $F(1,34)=1.14, M S E=.009, p=.31$; n.s., were indicated. However, a main effect of movement pattern occurred, $F(1,29)=10.76$, $M S E=.023, p<.004$, due to increased error rates for different movement patterns (i.e., observed and executed movements diverged) relative to same movement patterns $(11.95 \%, S E=1.8$ vs. $8.21 \%, S E=1.3$ ). There was also a significant main effect of occluder time, $F(2,58)=7.60, M S E=.011, p<.001$, indicating higher error rates for brief occluder durations of $100 \mathrm{~ms}(12.2 \%, S E=1.8)$ than longer durations ( $400 \mathrm{~ms}: 8.7 \%, S E=1.5 / 700 \mathrm{~ms}: 9.4 \%$, $S E=1.4)$.

To test our hypothesis that the degree of a representational overlap between observed and executed actions affected action prediction performance, we conducted a three-way repeated measures ANOVA including the within-subjects factors occluder time (100 vs. 400 vs. $700 \mathrm{~ms}$ ), pose time (400 vs. $700 \mathrm{~ms}$ ) and representational overlap (full vs. partial vs. no overlap). Most importantly, in line with our motor modulation hypothesis, a significant three-way interaction was revealed, $F(4,116)=2.54, M S E=.008$, $p<.04$. No further interactions were indicated. Again, there was no main effect of pose time, $F(1,29)=.46, M S E=.017, p=.50$; n.s., and a significant main effect of occluder time, $F(2,58)=6.39$, $M S E=.008, p<.003$. In addition, the factor representational overlap yielded a main effect, $F(2,58)=8.37, M S E=.014, p<.001$, due to lower error rates for 'full overlap' $(7.3 \%, S E=1.3)$ as compared to 'partial overlap' $(10.6 \%, S E=1.4)$ and 'no overlap' $(11.8 \%$, $S E=2.0$ ).

According to the real-time hypothesis, performance is predicted to be best when occluder time and pose time correspond to each other and deteriorates with increasing time distance between both factors (due to increased deviance of the actual test pose from the internal real-time model), thus producing a monotonic distance function. To test this effect, a three-way repeated measures ANOVA of the absolute time distances between occluder time and pose time (i.e., 0, 300, $600 \mathrm{~ms}$ ) was run, with the within-subjects factors distance ( 0 vs. 300 vs. 600 ms), body side (congruent vs. incongruent) and movement pattern (same vs. different) (cf. Experiment 1). It should be noted that positive and the negative time distances again did not differ from each other, $F(1,29)=.73$, MSE $=.015$, $p=.40$, n.s. In line with the real-time hypothesis, the error rates increased monotonically with increasing time distance between occluder time and pose time, yielding a marginal main effect of distance, $F(2,58)=3.01, M S E=.008, p=.07$, with a significant monotonic trend, $F(1,29)=4.41, M S E=.008, p<.05$, in the predicted way. There was a significant interaction of distance $\times$ body side $\times$ movement pattern, $F(2,58)=5.28, \quad M S E=.007, \quad p<.01$. Again, the distance factor neither interacted with body side, $F(2,58)=.61, M S E=.007, p=.54 ;$ n.s., nor movement pattern, $F(2,58)=.94, M S E=.005, p=.40$; n.s. Therefore, the results suggest that neither a correspondence in the body side nor in the movement pattern was, by itself, sufficient to influence the monotonic distance function (indicating real-time simulation).

Indeed, our hypothesis was that the degree of a representational overlap between executed and observed movements will vary the monotonic distance function. To test this hypothesis, a two-way ANOVA (repeated measures) with the within-subjects factors distance (0 vs. 300 vs. $600 \mathrm{~ms}$ ) and representational overlap (full vs. partial vs. no overlap) was run (cf. Experiment 1). Most importantly, in line with our hypothesis, a significant interaction between 'distance' and 'representational overlap' emerged, $F(4,116)=2.51, M S E=.005, p<.05$. Furthermore, there was a significant main effect of representational overlap, $F(2,58)=7.83$, $M S E=.008, p=.001$, due to fewer errors for 'full overlap' $(7.3 \%$, $S E=1.4)$ than 'partial overlap' $(11.0 \%, S E=1.5)$ and 'no overlap' $(12.1 \%, S E=2.1)$, while no main effect of distance was revealed, $F(2,58)=1.09, M S E=.006, p=.34 ;$ n.s.
Additional separate analyses of the 'representational overlap' conditions were conducted. In order to provide terminological consistency to Experiment 1, these conditions were again labelled according to the anatomical side of the body which was involved in the executed and observed movements. Hence, although one and the same label referred to different types of trials in both experiments due to the manipulation of the viewing direction (i.e., back view vs. front view), the label always described the mapping between the moved and observed anatomical side of the body. For example, while 'different body side/same movement pattern' implied a mirror-inverted constellation in Experiment 1 (i.e., front view), the identical term described a left-right reversed constellation in Experiment 2 (i.e., back view). However, in both trials, the executed and observed actions involved different anatomical body sides and the same movement pattern, which is directly and unambiguously expressed by the label 'different body side/ same movement pattern'.

In line with our motor modulation hypothesis, the results revealed a significant main effect of distance for the partial overlap condition, $F(2,58)=7.84, M S E=.004, p<.003$. This distance effect exhibited a significant linear trend, $F(1,29)=10.95, M S E=.049$, $p<.003$, in the predicted way (i.e., monotonic distance function) (Fig. 2B). In contrast, the conditions of full overlap $(F(2,58)=.37$, $M S E=.006, p=.69 ;$ n.s. $)$ and no overlap $(F(2,58)=.62, M S E=.007$, $p=.54$; n.s.) did not show this function. Hence, consistent with Experiment 1, a monotonic distance function (taken as evidence of internal simulation) emerged only when observed and executed movements shared common representational features, whereas the function was clearly absent for 'full overlap' and 'no overlap' conditions (Fig. 2B).

Analogous to the error rates, the RTs were analyzed using a four-way repeated measures ANOVA with the within-subjects factors occluder time (100 vs. 400 vs. $700 \mathrm{~ms}$ ), pose time (400 vs. $700 \mathrm{~ms}$ ), body side (congruent vs. incongruent) and movement pattern (same vs. different). Occluder time yielded a highly significant main effect, $F(2,58)=12.34, M S E=9030.58, p<.001$, due to higher RTs for short occlusion durations $(M=762.38 \mathrm{~ms}$, $S E=27.84)$ relative to longer occlusion durations $(400 \mathrm{~ms}$ : $M=723.90 \mathrm{~ms}, S E=24.12 / 700 \mathrm{~ms}: M=726.34 \mathrm{~ms}, S E=24.61)$ (cf. Experiment 1). Moreover, the main effect of movement pattern was significant, $F(1,29)=6.51, M S E=4199.87, p<.02$, due to higher RTs for different movement patterns $(M=743.70 \mathrm{~ms}, S E=24.40)$ than same movement pattern $(M=731.38 \mathrm{~ms}, S E=25.97)$. No further effects were indicated.

To test the RTs for a monotonic distance function, a three-way ANOVA (repeated measures) with the within-subjects factors distance ( 0 vs. 300 vs. $600 \mathrm{~ms}$ ), body side (congruent vs. incongruent) and movement pattern (same vs. different) was run. It revealed a significant main effect of distance, $F(2,58)=13.63, M S E=3751.86$, $p<.001$, with a significant linear trend, $F(1,29)=18.24, M S E=$ $4712.90, p<.001$ (i.e., monotonic distance function), as predicted by the real-time hypothesis (see Appendix A). An additional twoway ANOVA with the factors distance (0 vs. 300 vs. $600 \mathrm{~ms}$ ) and representational overlap (full vs. partial vs. no overlap) confirmed a main effect of distance, $F(2,58)=12.24, M S E=2707.10, p<.001$, with a linear trend, $F(1,29)=17.06, M S E=3310.23, p<.001$ (i.e., a monotonic distance function, reflecting increasing RTs with increasing time distances between occluder time and pose time). No further RT effects were obtained. Hence, no speed-accuracy trade offs were indicated (i.e., the RT pattern was not inverse to the error pattern).

These results provide evidence of our hypothesis that action execution affects internal action simulation (reflected by a monotonic distance function). In line with Experiment 1, our findings indicate that a representational overlap between observed and executed movements may be crucial for this influence. Moreover, 


\section{A. Experiment 1 (Front View)}
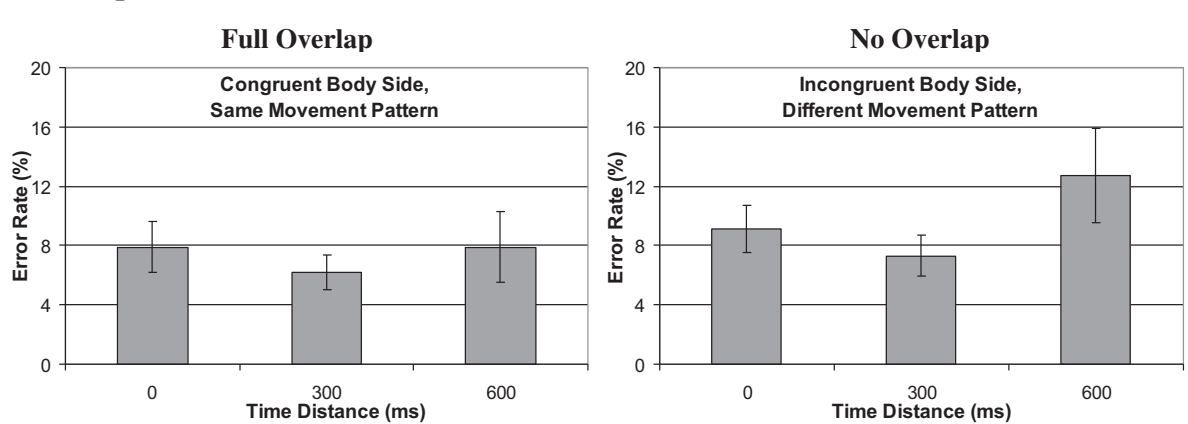

Partial Overlap
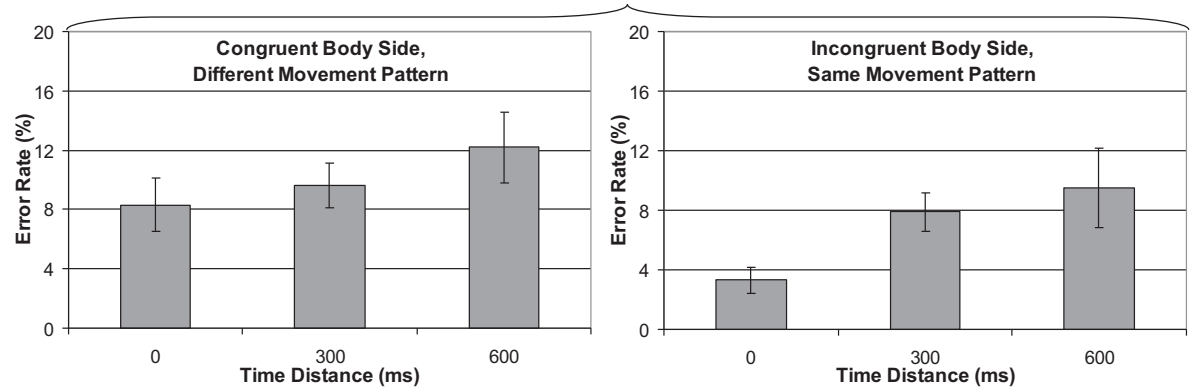

\section{B. Experiment 2 (Back View)}

Full Overlap

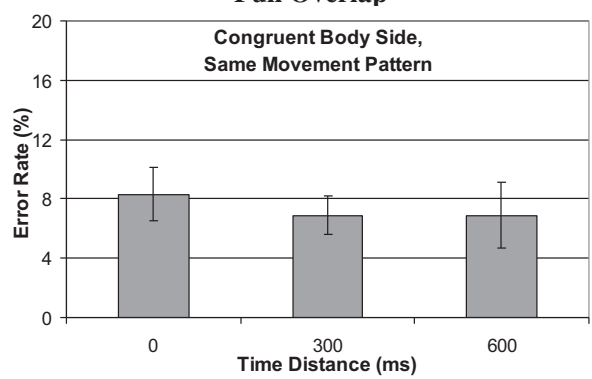

No Overlap

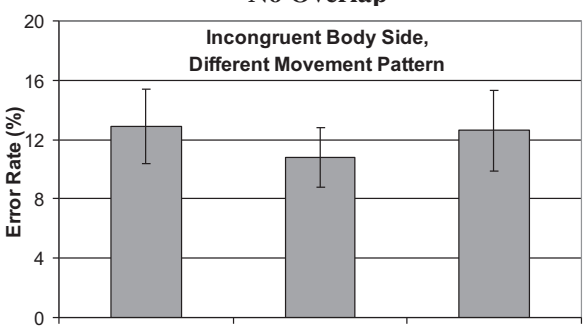

Time Distance (ms)

600

Partial Overlap
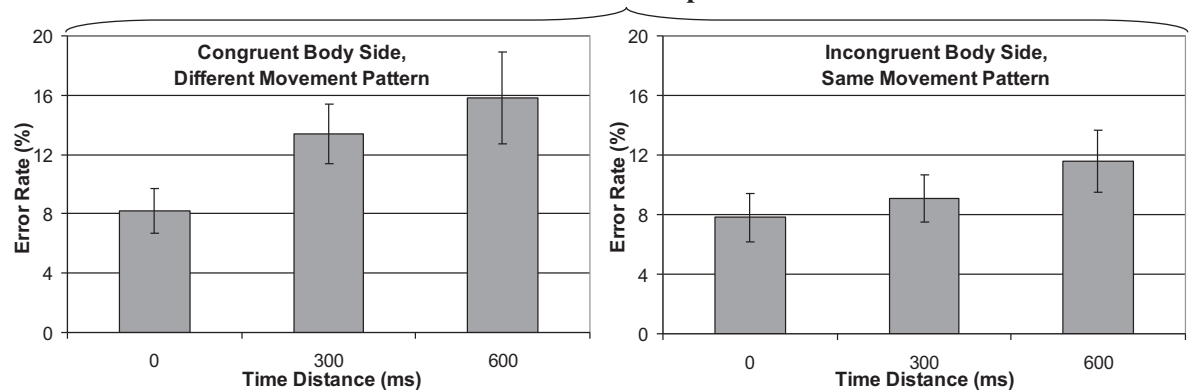

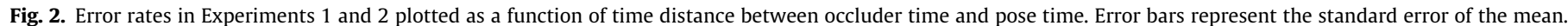

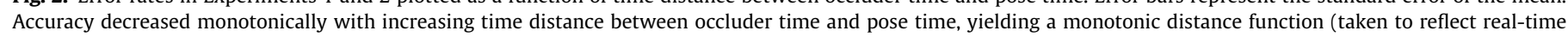

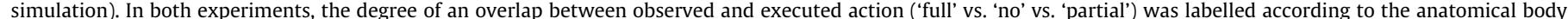

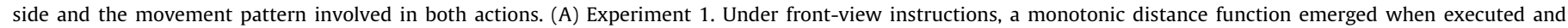

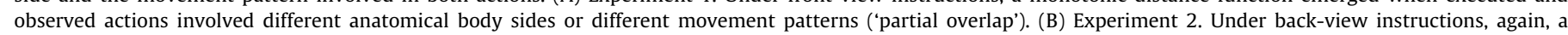

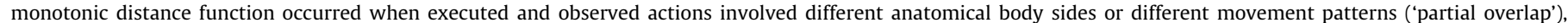
suggesting that anatomical mappings drive motor influences on action prediction performance (indicating action simulation).

the 'mirror-inverted' condition of Experiment 2 (implying spatial congruence between executed and observed movement due to back view instructions) clearly did not produce a monotonic distance function. Therefore, the present findings cannot be explained by pure spatial congruency effects.

\section{General discussion}

The results of the present study showed that action execution affected the performance in an action prediction task which is taken as an indication of internal real-time simulation (Graf et al., 
2007; Springer \& Prinz, 2010). This supports our assumption that motor processes are involved in action simulation.

In two experiments, our participants watched briefly occluded actions and judged whether the test pose presented after the occlusion depicted a continuation of the previous action sequence in the same or a slightly different depth angle (Graf et al., 2007). Response accuracy was best when the actions reappeared in a time-consistent manner and decreased with increasing time distance between the duration of occlusion (=occluder time) and the temporal advance of the test pose shown after occlusion (=pose time) (i.e., monotonic distance function). This function, which can be derived from an occluder time $\times$ pose time interaction, is in line with previous findings showing that action prediction involves internal models that run in real time to the observed action (Graf et al., 2007; cf. Flanagan \& Johansson, 2003; Verfaillie \& Daems, 2002). Performance was best when occluder time and pose time matched because the internal model of the last-seen action pose prior to occlusion was internally updated in real time, thus matching the actual test pose. Furthermore, performance decreased with increasing time distance due to an increasing difference between the real-time model and the actual test pose. This pattern was indicated although explicit judgements about the timing of the observed actions were not required; even more, participants were instructed to decide whether the stimulus was a continuation of the movement at any point in time. This implicit measurement rules out a potential alternative explanation that the effects were merely due to task strategies.

\subsection{Motor influences on action prediction performance}

In both experiments, action prediction performance (i.e., the monotonic distance function) was clearly influenced by a secondary execution task. The observed and executed arm movements were either congruent or incongruent regarding the involved movement pattern (upwards vs. sideways) and the anatomical body side (left vs. right), resulting in the conditions of 'full', 'partial' and 'no' representational overlap between observed and executed movements. While there was no influence of anatomical body side or movement pattern per se, the monotonic distance function was varied by the degree of a representational overlap. More specifically, the function (indicating internal action simulation) was obtained for a 'partial overlap' (implying congruence in the anatomical body sides used or in the exact movement patterns involved) while it was absent in the 'full' and 'no' overlap conditions (i.e., full correspondence or no correspondence in both dimensions, respectively). This pattern was obtained in both experiments, independent of the instructions given about the viewing direction (i.e., front view vs. back view of the observed actor).

In sum, these findings suggest that the observed motor influence on action prediction performance depends on the degree of an overlap between executed and observed action. This is in line with our motor modulation hypothesis.

In Experiment 1, the partial overlap condition included a 'mirror-inverted' constellation (i.e., different body side, same movement pattern). As the participants were instructed that they would see the PL actor from the front, these trials additionally implied spatial congruence between executed and observed movements (i.e., both occurring on the same side of the screen). Therefore, one may argue that it is merely spatial congruence that drives the observed motor influence (Craighero et al., 2002; Christensen et al., 2009; Kilner et al., 2009).

Nevertheless, Experiment 2 clearly contradicted a purely spatial account of the present data. As the participants were instructed that they would see the PL actor from behind, spatial congruence converged with anatomical congruence between observed and executed actions. Under this viewing condition, again, a pronounced monotonic distance function (indicating real-time simulation) emerged when executed and observed actions shared common representational features ('partial overlap'). In contrast to Experiment 1 , however, the 'mirror-inverted' constellation (implying spatial congruence between executed and observed movements) did not show the monotonic distance function. Therefore, spatial congruence alone cannot be a sufficient explanation for the present data. The findings suggest that the degree of a representational overlap between executed and observed movement matters, as defined by the anatomical side of the body and the movement pattern involved. The accuracy with which observers predict forthcoming actions seems to depend on anatomical mappings between their own action and the observed action (Gillmeister, Catmur, Liepelt, Brass, \& Heyes, 2008; Liepelt, Prinz, \& Brass, 2010; Sambrook, 1998; Wapner \& Cirillo, 1968).

This view was further substantiated by an additional overall analysis run across both experiments including 'viewing direction' (front vs. back view) as a between subjects factor. Because of the fact that the factors anatomical body side and movement pattern, by themselves, did not produce an effect on the monotonic distance function, a three-way repeated measures ANOVA including the factors distance ( 0 vs. 300 vs. $600 \mathrm{~ms}$ ), representational overlap (full vs. partial vs. no overlap) and viewing direction (front vs. back view) was used. The results revealed a significant monotonic distance function, $F(1,63)=4.35, M S E=.010, p<.04$, in line with the real-time hypothesis. Moreover, the distance factor interacted with the representational overlap factor, $F(4,252)=4,66, M S E=.022$, $p<.002$. While the 'partial overlap' condition revealed a monotonic distance function, $F(1,63)=22.19$, MSE $=.004, p<.001$, the function was absent for the 'full overlap' and 'no overlap' conditions (cf. Fig. 2).

Interestingly, no statistical effects of viewing direction were obtained, indicating that the front view and the back view instructions did not lead to general performance differences. Both experiments included exactly the same point-light actions and differed only in the viewing direction instructions. Consistent with our results of an independent rating of the PL stimuli (as being spontaneously perceived from the front vs. from the back; cf. Experiment 2), this finding suggests that the front view and the back view on the (identical) PL stimuli were equally plausible and were effectively changed by our instructions regarding the viewing direction.

Moreover, the factor 'representational overlap' yielded a significant main effect, $F(2,126)=9.43, M S E=.008, p<.001$, indicating less errors when executed and observed movement fully corresponded $(7.3 \%, S E=1.1)$ as compared to the partial overlap $(9.6 \%$, $S E=.94)$ and no overlap condition $(10.8 \%, S E=1.4)$. This finding suggests that in the case of a full overlap between executed and observed actions (i.e., same anatomical body side, same movement pattern), the participants were able to solve the task effectively, although they did not show a monotonic distance function (taken to reflect internal simulation). This is in line with our motor modulation hypothesis.

Corresponding to the initially cited theory of event coding (TEC; Hommel et al., 2001), the present findings suggest that the extent to which executed and observed actions draw on shared representational codes modulate the extent to which an internal simulation process is recruited during action prediction. According to the notion of 'common coding' (Hommel et al., 2001; Prinz, 1997), the same underlying representations are involved when executing an action and observing the same action in another individual. Accordingly, participants may use the same motor representations activated during action execution to predict the future course of a corresponding action observed in other individuals (Knoblich, Seigerschmidt, Flach, \& Prinz, 2002; Wilson \& Knoblich, 2005). If so, the requirement to internally simulate an observed action 
should be reduced when executed and observed actions fully correspond. Given a 'full overlap' in terms of anatomical body sides and movement patterns, the executed action itself already provides a continuously updated internal reference by which the participants can effectively solve the task. As a result, our participants were able to solve the prediction task effectively, without the need for an internal simulation (because the reference information against which the test pose is matched can be determined from the executed motor movement itself and therefore does not require an additional simulation process). Hence, no simulation process was indicated.

Conversely, in the 'no overlap' condition, the motor representations used for motor execution may have not (or to a lesser degree) been accessible to an internal update of the observed action. Given a complete lack of correspondence between executed and observed actions, execution seems to strongly interfere with internal simulation (Prinz, 1997; Wilson \& Knoblich, 2005), implying that an internal simulation process has to be shielded from the information available from executing a movement which is different from the observed movement in two dimensions. Due to this interference, internal simulation is highly costly, error-prone and, hence, inefficient. Correspondingly, we did not find evidence of internal simulation (i.e., no distance function was indicated). In this condition, we found substantially increased error rates, suggesting that interference impeded internal simulation during action prediction.

In contrast, given partial correspondence between perception and action, a pronounced monotonic distance function emerged, providing evidence of internal real-time simulation. Here, realtime simulation processes may have effectively been used for solving the task. Given a partial representational overlap, the cost/benefit relation for running internal simulations was more balanced, and simulation was partially efficient. In line with this view, it has been shown that observers are able to predict not only the consequences of their own actions but also the consequence of others' actions (e.g., Sato, 2008), indicating that the temporal predictions generated by one's own motor system can also be applied to predict actions observed in others (Blakemore \& Frith, 2005; Kilner et al., 2007).

Notably, concurrent motor execution did not prevent participants from solving the task (i.e., prediction performance was consistently above chance level). Whether they may have used additional processes in the 'full overlap' and 'no overlap' conditions, cannot be elucidated by the present data. Action prediction may rely on processes that are less motor-based when one's own motor representations are constrained by action execution. Likewise, perception biases associated with motor simulations (e.g., judging objects as nearer to the body than they actually are) have been shown to disappear when participants concurrently performed actions requiring the same effector as reaching for the object (Witt \& Proffitt, 2008).

\subsection{Summary and implications}

The present data provide evidence of internal simulation during action prediction (as indicated by a monotonic distance function) when observed and simultaneously performed actions involved the same anatomical body side or the same movement pattern (i.e., partial overlap). In contrast, we found no evidence of internal simulation when both actions were fully congruent (i.e., same movement patterns, same anatomical body sides) or fully incongruent (i.e., different movement patterns, different anatomical body sides). These findings suggest that motor processes are involved in action prediction. The size of their impact on action prediction performance seems to depend on the anatomical mapping between executed and observed actions. In line with this view, the results showed that the viewing direction (i.e., whether the observed actor was perceived from the front or the back) did not interact with any other critical factor. This suggests that the observed motor modulation of action prediction performance was not only due to the congruence of the spatial mappings between executed and observed actions, but at least in part due to their anatomical correspondence.

The present findings are in line with previous evidence showing that action observation activates the motor system in corresponding somatotopic organization (Buccino et al., 2001; Decety \& Grèzes, 1999, 2006; Grèzes \& Decety, 2001). Moreover, our findings correspond to the notion that a structural representation of the human body is allocentric (i.e., not tied to the observer's viewpoint) and can therefore be mapped isomorphically onto external information about another individual's body (Wilson \& Knoblich, 2005).

\section{Acknowledgment}

We thank Mathias Lesche for experimental programming.

\section{Appendix A. Supplementary data}

Supplementary data associated with this article can be found, in the online version, at doi:10.1016/j.bandc.2011.03.007.

\section{References}

Bertelson, P., \& Tisseyre, F. (1968). The time course of preparation with regular and irregular foreperiods. Quarterly Journal of Experimental Psychology, 20, 297-300. Blakemore, S. J., \& Frith, C. (2005). The role of motor contagion in the prediction of action. Neuropsychologia, 43, 260-267.

Brass, M., Bekkering, H., \& Prinz, W. (2001). Movement observation affects movement execution in a simple response task. Acta Psychologica, 106, 3-22.

Buccino, G., Binkofski, F., Fink, G. R., Fadiga, L., Fogassi, L., Gallese, V., et al. (2001) Action observation activates premotor and parietal areas in a somatotopic manner: An fMRI study. European Journal of Neuroscience, 13, 400-404.

Craighero, L., Bello, A., Fadiga, L., \& Rizzolatti, G. (2002). Hand action preparation influences the responses to hand pictures. Neuropsychologia, 40, 492-502.

Christensen, A., Ilg, W., Karnath, H.-O., \& Giese, M. A. (2009). Influence of spatial and temporal congruency between executed and observed movements on the recognition of biological motion. Journal of Vision, 9(8), 614. doi:10.1167/ 9.8.614.

Decety, J., \& Grèzes, J. (2006). The power of simulation: Imagining one's own and other's behavior. Brain Research, 1079, 4-14.

Decety, J., \& Grèzes, J. (1999). Neural mechanisms subserving the perception of human actions. Trends in Cognitive Sciences, 3, 172-178.

Dinstein, I., Hasson, U., Rubin, N., \& Heeger, D. J. (2007). Brain areas selective for both observed and executed movements. Journal of Neurophysiology, 98, 1415-1427.

Flanagan, J. R., \& Johansson, R. S. (2003). Action plans used in action observation. Nature, 424, 769-771.

Gallese, V. (2005). Embodied simulation: From neurons to phenomenal experience. Phenomenology and the Cognitive Sciences, 4, 23-48.

Gallese, V., Fadiga, L., Fogassi, L., \& Rizzolatti, G. (1996). Action recognition in the premotor cortex. Brain, 119, 593-609.

Gillmeister, H., Catmur, C., Liepelt, R., Brass, M., \& Heyes, C. (2008). Experiencebased priming of body parts: A study of action imitation. Brain Research, 1217, 157-170.

Graf, M., Reitzner, B., Corves, C., Casile, A., Giese, M., \& Prinz, W. (2007). Predicting point-light actions in real-time. NeuroImage, 36, T22-T32.

Grèzes, J., \& Decety, J. (2001). Functional anatomy of execution, mental simulation, observation, and verb generation of actions: A meta-analysis. Human Brain Mapping, 12, 1-19.

Grush, R. (2004). The emulation theory of representation: Motor control, imagery, and perception. Behavioural Brain Science, 27, 377-442.

Hamilton, A., Wolpert, D., \& Frith, U. (2004). Your own action influences how you perceive another person's action. Current Biology, 14, 493-498.

Hommel, B., Müsseler, J., Aschersleben, G., \& Prinz, W. (2001). Aschersleben, G., \& Prinz, W. (2001). The theory of event coding (TEC). A framework for perception and action planning. Behavioural and Brain Sciences, 24, 849-937.

Huynh, H., \& Feldt, L. S. (1970). Conditions under which the mean square ratios in repeated measurement designs have exact F-distributions. Journal of the American Statistical Association, 65, 1582-1589.

Iacoboni, M., \& Dapretto, M. (2006). The mirror neuron system and the consequences of its dysfunction. Nature reviews. Neuroscience, 7, 942.

Jeannerod, M. (2001). Neural simulation of action: A unifying mechanism for motor cognition. Neurolmage, 14, S103-S109.

Johansson, G. (1973). Visual perception of biological motion and a model for its analysis. Perception and Psychophysics, 14, 201-211. 
Kilner, J. M., Friston, K. J., \& Frith, C. D. (2007). Predictive coding: An account of the mirror neuron system. Cognitive Process, 8, 159-166.

Kilner, J. M., Marchant, J. L., \& Frith, C. D. (2009). Relationship between activity in human primary motor cortex during action observation and the mirror neuron system. Plosone, 4, 1-10.

Kilner, J. M., Paulignan, Y., \& Blakemore, S.-J. (2003). An interference effect of observed biological movement on action. Current Biology, 13, 522-525.

Kilner, J. M., Vargas, C., Duval, S., Blakemore, S. J., \& Sirigu, A. (2004). Motor activation prior to observation of a predicted movement. Nature Neuroscience, 7 , 1299-1301.

Knoblich, G., Seigerschmidt, E., Flach, R., \& Prinz, W. (2002). Authorship effects in the prediction of handwriting strokes: Evidence for action simulation during action perception. The Quarterly Journal of Experimental Psychology, 55, 1027-1046.

Liepelt, R., Prinz, W., \& Brass, M. (2010). When do we simulate non-human agents? Dissociating communicative and non-communicative actions. Cognition, 115(3), 426-434.

Liepelt, R., von Cramon, D. Y., \& Brass, M. (2008). What is matched in direct matching? Intention attribution modulates motor priming. Journal of Experimental Psychology: Human Perception and Performance, 34(3), 578-591.

Marconi, B., Pecchioli, C., Koch, G., \& Caltagirone, C. (2007). Functional overlap between hand and forearm motor cortical representations during motor cognitive tasks. Clinical Neurophysiology, 118, 1767-1775.

Oldfield, R. C. (1971). Assessment and analysis of handedness - Edinburgh inventory. Neuropsychologia, 9, 97-113.

Prinz, W. (1997). Perception and action planning. European Journal of Cognitive Psychology, 9, 129-154.

Prinz, W. (1990). A common coding approach to perception and action. In O. Neumann \& W. Prinz (Eds.), Relationships between perception and action: Current approaches (pp. 167-201). Berlin: Springer.

Sambrook, T. D. (1998). Does visual perspective matter in imitation? Perception, 27, 1461-1473.
Sanders, A. F. (1998). Elements of human performance: Reaction processes and attention in human skill. Mahwah, NJ: Lawrence Erlbaum.

Sato, A. (2008). Action observation modulates auditory perception of the consequences of others' actions. Consciousness and Cognition, 17, 1219-1227.

Schütz-Bosbach, S., \& Prinz, W. (2007). Perceptual resonance: Action-induced modulation of perception. Trends in Cognitive Sciences, 11(8), 349-355.

Sebanz, N., \& Knoblich, G. (2009). Prediction in joint action: What, when, and where Topics in Cognitive Science, 1(2), 353-367.

Springer, A., \& Prinz, W. (2010). Action semantics modulate action prediction. The Quarterly Journal of Experimental Psychology, 63(11), 2141-2158.

Stadler, W., Schubotz, R., von Cramon, D. Y., Springer, A., Graf, M., \& Prinz, W. (in press). Predicting and memorizing observed action: Differential premotor cortex involvement. Human Brain Mapping, doi:10.1002/hbm.20949.

Thornton, I. M., \& Knoblich, G. (2006). Action perception: Seeing the world through a moving body. Current Biology, 16(1), R27-R29.

Verfaillie, K., \& Daems, A. (2002). Representing and anticipating human actions in vision. Visual Cognition, 9, 217-232.

Wapner, S., \& Cirillo, L. (1968). Imitation of a model's hand movements: Age changes in transposition of left-right relations. Child Development, 39, 887-894.

Wilson, M., \& Knoblich, G. (2005). The case for motor involvement in perceiving conspecifics. Psychological Bulletin, 131, 460-473.

Witt, J. K., \& Proffitt, D. R. (2008). Action-specific influences on distance perception: A role for motor simulation. Journal of Experimental Psychology: Human Perception and Performance, 34, 1479-1492.

Wolpert, D. M., \& Flanagan, J. R. (2001). Motor prediction. Current Biology, 11 R729-R732.

Zwickel, J., Grosjean, M., \& Prinz, W. (2007). Seeing while moving: Measuring the online influence of action on perception. The Quarterly Journal of Experimental Psychology, 60(8), 1063-1071. 